Originalveröffentlichung in: H. Gebhardt, H. Kiesel (Hg.), Weltbilder, Heidelberger Jahrbücher 47, 2003, S. $97-110$

\title{
Das Band zwischen allen Dingen - Wissenskultur und Weltbild im Alten Orient
}

STEFAN M. MAUL

\section{I}

Die Kunst des Schreibers ist die Mutter derer, die lesen, und der Vater der Gelehrten.

Die Kunst des Schreibers bringt Freude, aber niemals kann man sich an ihr sättigen;

Die Kunst des Schreibers ist zwar nicht einfach zu erlernen, aber der, der sie erlernt, fürchtet sie nicht.

Bemühe dich um die Kunst des Schreibers, und sie wird dich bereichern.

Sei arbeitsam in der Kunst des Schreibers, und sie wird dich auch mit Gütern versorgen.

Sei der Kunst des Schreibers gegenüber nicht gleichgültig, lasse den Arm nicht ruhen: denn die Kunst des Schreibers ist ein "Haus der Schönheit", das dir die Weisheit des Enki eröffnet.

Wenn du dich ihr unermüdlich widmest, wird sie dir ihre Geheimnisse enthüllen.

Nur wenn du ihr gegenüber gleichgültig bist, wird man Schlechtes über dich sagen.

Die Kunst des Schreibers ist ein gutes Los, das auch Reichtum und Überfluss bringt.

Wenn du noch jung bist [und lernst], dann stöhnst du, bist du aber groß, dann ist sie [ein Vergnügen].

Die Kunst des Schreibers ist das Band zwischen allen Dingen. ${ }^{1}$

Ein jeder, der beseelt ist von seiner Wissenschaft, der gerungen hat um Erkenntnis, der das Verschlungene zu entwirren versucht und nach langem Mühen eine Erklärung für das zuvor Unverständliche gefunden hat, wird die tiefe Freude an Einsicht kennen, der in diesem Preislied auf $n$ a $\mathrm{m}$ - $\mathrm{d} u \mathrm{~b}$ - $\mathrm{s}$ a r,

${ }^{1}$ Die Übersetzung aus dem Sumerischen richtet sich nach der Edition von Sjöberg, In Praise of the Scribal Art. 
die Kunst des (Tontafel-)Schreibers, Ausdruck verliehen ist. In den Skriptorien der berühmten Bibliothek des assyrischen Königs Assurbanipal (668-631 v. Chr.) zu Ninive wurde dieser sumerische Text, der vielleicht schon vor nahezu 4000 Jahren entstand, gleich mehrfach abgeschrieben. Auch den Fachfremden lässt er erahnen, dass ein tieferes Verständnis der Geisteswelt des Alten Orients, dessen schriftliche und archäologische Quellen auf den ersten Blick oft fremd und wenig gefällig wirken, durchaus möglich, vor allem aber lohnenswert ist. Das Gedicht lässt keinen Zweifel daran, dass auch im Alten Orient Studium und Gelehrsamkeit dem forschenden Geist dieselbe Ernsthaftigkeit, Selbstdisziplin und Begeisterungsfähigkeit abverlangte, die auch heute noch unabdingbare Voraussetzungen für jede gute, seriöse Wissenschaft sind. In den Schreibstuben Assurbanipals, der in seiner Palastbibliothek das gesamte Wissen seiner Zeit zusammentragen wollte, wirkten wohl nur die fähigsten Gelehrten Assyriens und Babyloniens. Bereits ein oberflächliches Studium der Bibliothekstafeln, die man in den Ruinen des Königspalastes in Ninive fand, stellt dies eindrucksvoll unter Beweis. Die elegant geformten, mit einer ebenmäßigen, geradezu genormten neuassyrischen Keilschrift beschriebenen Tontafeln zählen schon äußerlich zu den schönsten Schriftstücken, die der Alte Orient hervorgebracht hat. Betrachtet man sie genauer, stellt man fest, dass sie das Produkt einer wahrhaft wissenschaftlich zu nennenden philologischen Recherche sind. Zur Erstellung der Editionen von literarischen, religiösen, medizinischen und divinatorischen Texten wurden zahlreiche Textvertreter aus allen Landesteilen herangezogen und unter größter Sachkenntnis stets die beste Textvariante ausgewählt. Einmalig in der Philologie alter Kulturen dürfte der Umstand sein, dass inhaltliche Abweichungen von Textzeugen in einer Art Variantenapparat verzeichnet und die Stellen, die in den Textvorlagen zerstört waren, auch dann in den neuen Ausgaben als Textlücke gekennzeichnet wurden, wenn die Ergänzung des Textes auf der Hand lag. Eine solche philologisch-pedantische Arbeitsweise unterscheidet sich von der Tätigkeit des modernen Assyriologen nur geringfügig. Mit der gleichen Gewissenhaftigkeit wurden in den Schreibstuben des Königspalastes Gebete, Ritualbeschreibungen und exorzistische Fachliteratur, medizinische Texte und Omensammlungen unterschiedlichster Provenienz gesammelt, gesichtet und in Kompendien, Serien und Handbüchern zusammengefasst, die von da an bis zum Ausklingen der Keilschriftkultur im 1. Jh. n. Chr. als kanonisch angesehen wurden. Darüber hinaus legten gelehrte Schreiber - modernen Assyriologen gleich - paläographische Zeichenlisten an, die im Elementarunterricht Verwendung fanden und bereits den jugendlichen Keilschriftstudenten ein Mittel in die Hand gaben, eigenständig die auch damals stets bei Ausschachtungsarbeiten gefundenen Schriftzeugnisse älterer Perioden zu erschließen. An dem Hofe neuassyrischer Könige kursierten sogar Keilschriftzeichenlisten, die synoptisch die zeitgenössischen neuassyrischen Zeichenformen den noch deutlich bildhaften Zeichenformen der Jahr- 
tausende zurückliegenden Zeit der Schrifterfindung gegenüberstellten. Wenige Tafelbruchstücke zeugen sogar davon, dass neuassyrische Schreiber sich darin versuchten, Texte in diesem urtümlichen Duktus zu verfassen. Daher muss die auf den ersten Blick prahlerische Angabe Assurbanipals, er habe gar "Steine aus den Zeiten vor der Flut" lesen können, ernst genommen werden, zumal sich die Abschrift einer zweisprachigen Schülerfibel erhalten hat, die eigens für das Studium des damals noch jungen Prinzen Assurbanipal geschrieben worden war. Wie dem auch sei, die paläographischen und sprachhistorischen Kenntnisse neuassyrischer und neubabylonischer Schreiber waren durch stetes Studium derart perfektioniert, dass sie offenbar mit Leichtigkeit sowohl Faksimiles als auch Neuschöpfungen von Schriftstücken in der Machart des 3. und 2. Jahrtausends v. Chr. herstellen konnten, die so formvollendet erscheinen, dass auch moderne Assyriologen noch über das wahre Alter der Dokumente streiten.

Das Interesse altorientalischer Schreiber an der Schriftgeschichte ihrer Kultur war nicht unmotiviert. Im Auftrage der Könige fertigten sie Inschriften, niedergeschrieben in altertümlichen Keilschriftzeichenformen, die im zeitgenössischen Alltagsleben schon mehr als anderthalb Jahrtausende außer Gebrauch waren. Die Schreiber und Gelehrten bedienten sich auch einer babylonischen Kunstsprache, die sich - weit entfernt von der zeitgenössischen Sprache des Alltags - an der altertümlichen, als klassisch empfundenen akkadischen Sprache orientierte, die zu Beginn des 2. Jahrtausends v. Chr. gesprochen wurden, und pflegten unbeirrt das bereits um $2000 \mathrm{v}$. Chr. als gesprochene Sprache ausgestorbene Sumerische. Dies zeigt, welch' mächtige, alles durchdringende normative Kraft das Althergebrachte in den Kulturen des Alten Orients besaß, und wie man durch das Wiederbeleben der Formen des eigenen, Altertums', die Zeiten überbrückend, nach der klaren uranfänglichen Ordnung der,fernen Zeit'strebte, der die Götter selbst im Schöpfungsakt ihre Gestalt gegeben hatten. ${ }^{2}$

Obgleich nicht zuletzt die Gelehrten am Hofe Assurbanipals, die Zeugen einer nie zuvor gesehenen Machtentfaltung des assyrischen Reiches wurden, aus engster Nähe sahen, welche „Fortschritte" astronomische Beobachtungsformen und technische Entwicklungen machten, und das Phänomen der Kulturentwicklung am Beispiel ihrer paläographischen Studien genau beobachteten, war ihr Bild von Wissenschaft und Erkenntnis weit weniger als das unsere von einem Fortschrittsgedanken geprägt. Sie lehrten, dass das gesamte Wissen bereits im Uranfang der Welt den Menschen an die Hand gegeben

${ }^{2}$ Hierzu vgl. auch Maul in: Kuhn/Stahl (Hrsg.), Die Gegenwart des Altertums, S. 117-124. 
worden war. Sämtliche kulturellen Errungenschaften, auch die neuesten der Gegenwart, seien es die Fertigkeiten und Techniken der Baukunst, die Kunst der Schreiber, Goldschmiede und Schreiner sowie alle weiteren Technologien galten ihnen als Offenbarungen der Götter, die diese den Menschen zum Anbeginn der Zeiten geschenkt hatten. Noch Berossos, ein Marduk-Priester des 3. Jh. v. Chr., der mit seinem griechischsprachigen Werk Babyloniaka ${ }^{3}$ der hellenistischen Welt Geschichte und Kultur des alten Babyloniens nahe brachte, hielt dieses Selbstverständnis der babylonischen Gelehrsamkeit für wesentlich: Ein fischgestaltiges Wesen namens Oannes sei, so Berossos, im ersten Jahr der Welt, also unmittelbar nach Erschaffung von Himmel, Erde und Menschen, aus dem persischen Golf gestiegen und habe „die Menschen die Schriftkunde und die mannigfaltigen Verfahrungsweisen der Künste, die Bildungen von Städten und die Gründungen von Tempeln, auch der Gesetze Handhabung, die Geometrie und die Rechenkünste, sowie das Einbringen von Saatgut und Früchten [gelehrt], was nur immer der Häuslichkeit des Lebens der Welt zustatten kommt, überlieferte es (d.h. das „Tier“ [ $\tau o ́ \zeta(\dot{o} \nu$ ] Oannes) den Menschen; und seit jener Zeit werde von keinem anderen mehr etwas erfunden". ${ }^{4}$ Ferner habe Oannes über das Werden der Welt und das Staatswesen geschrieben und den Menschen Sprache und Kunstfertigkeit verliehen. ${ }^{5}$

Auch in Keilschriftquellen selbst lässt sich die Vorstellung nachweisen, dass Wissen und Künste nicht als menschliche Errungenschaften galten. Das Wirken der Gelehrten bestand in diesem Selbstverständnis darin, jeweils in ihrer Zeit das offenbarte Wissen zu durchdringen, zu bewahren und zum Wohle der Welt anzuwenden. Im folgenden Text wird dies für die Verfahren der Divination geschildert, welche die Götter Enmeduranki, einem vorsintflutlichen König von Sippar, offenbarten:

„Den Enmeduranki, den König der Stadt Sippar, den Liebling [der Götter] Anu, Enlil und Ea, ernannte [der Sonnengott] Schamasch im [Tempel] Ebabbar. Schamasch und Adad $^{6}$ ließen ihn in ihrer Versammlung Platz nehmen und Schamasch und Adad ehrten ihn. Schamasch und Adad ließen ihn vor sich Platz nehmen auf einem goldenen Thron. Sie ließen ihn sehen [die Kunst], das ... in Wasser zu betrachten, das Geheimnis von Anu, Enlil und Ea. Die Tafel der Götter, die Leber, das Geheimnis von Himmel und Erde, gaben sie ihm [...] Und er ließ, gemäß ihrem Ausspruch, die Söhne [der Städte] Nippur, Sippar und Babylon vor sich eintreten und ehrte sie. Auf Thronen ließ er sie vor sich Platz nehmen. Er ließ sie sehen [die Kunst], das Öl in Wasser zu betrachten, das Geheimnis von Anu, Enlil und Ea. Die Tafel der Götter, die

\footnotetext{
Siehe Schnabel, Berossos und die babylonisch-hellenistische Literatur, und die Übersetzung: Burstein, The Babyloniaca of Berossus.

" Vgl. Schnabel, Berossos und die babylonisch-hellenistische Literatur, S. 253.

Ebd., S. 254.

"Sonnen- und Wettergott.
} 
Leber, das Geheimnis von Himmel und Erde, gaben er ihnen [...]. Was die Tafel der Götter, die Leber, das Geheimnis von Himmel und Erde, und [die Kunst] anbetrifft, das Öl in Wasser zu betrachten, das Geheimnis von Anu, Enlil und Ea und das, was in den Kommentaren steht, was Enuma Anu Enlil ${ }^{7}$ anbetrifft und die Kunst, Multiplikationen zu errechnen: Der wissende Gelehrte, der das Geheimnis der großen Götter bewahrt, möge seinen Sohn, den er liebt, vereidigen mit Tafel und Griffel und ihn lernen lassen. ${ }^{8}$

Es erscheint nur folgerichtig, dass im mesopotamischen Sintflutmythos Uta-napischti, der babylonische Noah, nicht nur die Lebewesen in seine Arche lud, um sie in die neue Zeit zu retten, sondern auch dafür sorgte, dass das Wissen und die Künste das göttliche Weltengericht überdauerten. Im Gilgamesch-Epos berichtet der Sintflutheld:

Alles, was mir zur Verfügung stand, lud ich ein.

Alles, was mir zur Verfügung stand an Silber, lud ich ein.

Alles, was mir zur Verfügung stand an Gold, lud ich ein.

Alles, was mir zur Verfügung stand an jeglichem Samen des Lebens, lud ich ein.

An Bord des Schiffes ließ ich steigen meine gesamte Familie und meine Sippe. Das Vieh der Steppe, das Getier der Steppe [und] die Vertreter jeglicher Künste ließ ich einsteigen.?

Berossos weiß gar zu berichten, dass der Weisheitsgott der babylonischen Noah-Gestalt den Auftrag erteilte „die ersten, die mittleren und die letzten Schriftwerke" zu vergraben und so vor ihrem Untergang zu retten. Nach der Flut sollten sie dann wieder ausgegraben ${ }^{10}$ und "der Menschheit übergeben “ werden. $^{11}$

Für den mesopotamischen Menschen ist, abweichend von unserem Wissenschaftsverständnis, so Erkenntnisgewinn und „wissenschaftlicher Fortschritt" nicht in erster Linie "Neues“, sondern ein aktives (durchaus mit persönlicher Leistung verbundenes) Näherrücken an die im Uranfang gegebene und stets vom Menschen zu durchdringende göttliche Offenbarung. Es ist nur folgerichtig, dass - anders als in der griechisch-hellenistischen Kultur, in deren Tradition wir uns sehen - in Mesopotamien Erkenntnisse, Erfindungen und Neuschöpfungen in der Regel nicht mit dem Namen von Menschen, sondern mit dem von Göttern in Verbindung gebracht werden.

\footnotetext{
7 Enuma Anu Enlil wurde das sehr umfangreiche keilschriftliche Werk genannt, in dem die Gesetzmäßigkeiten der Astrologie niedergelegt waren.

${ }^{8}$ Lambert, The Qualifications of Babylonian Diviners, S. 148.

9 Gilg. XI, 81-86.

${ }^{10}$ Zweifelsfrei verbirgt sich hinter dieser Erzählung eine Ätiologie für den Umstand, dass man in den alten Städten Mesopotamiens bei Bauarbeiten immer wieder auf uralte Schriftzeugnisse stieß.

${ }^{11}$ Siehe Schnabel, Berossos und die babylonisch-hellenistische Literatur, S. 264f., und Burstein, The Babyloniaca of Berossus.
} 


\section{III}

Betrachten wir das Schrifttum der bereits im 2. Jahrtausend v. Chr. hochentwickelten mesopotamischen Mathematik, sehen wir babylonische Gelehrte komplexe Gleichungen 1. und 2. Grades lösen, mit einem Näherungswert der Zahl $\pi$ operieren und mathematische Probleme lösen, welche die Kenntnis des „Satzes des Pythagoras“ vorauszusetzen scheinen. Gleichwohl finden sich in dem mathematischen Texten Mesopotamiens weder Beweisführungen noch explizit formulierte mathematische Gesetze. Vergleichbares gilt für die Geometrie und die beobachtende und rechnende Astronomie. Wenn Astronomiehistoriker von „babylonischen Mond- oder Planetentheorien sprechen“, so schreibt W. von Soden, „so meinen sie nirgendwo formulierte Auffassungen, die man aus den sehr komplizierten Zahlenreihen der Texte ablesen kann und die die Griechen als eine Theorie hätten formulieren können. ${ }^{\text {"12 }}$ Der sich in diesem Befund offenbarende Mangel an Theorie und Abstraktionsvermögen, so sind sich die heutigen Wissenschaftshistoriker einig, habe dazu geführt, dass sich in Mesopotamien "trotz zahlreicher guter Beobachtungen und Berechnungen keine echte Wissenschaft ${ }^{{ }^{\prime 13}}$ habe entwickeln können. Diese aus der Perspektive unseres heutigen Wissenschaftsverständnisses getroffene Wertung, die so durchaus ihre Berechtigung haben mag, möchte ich hier nicht weiterverfolgen. Denn sie ist kaum hilfreich, wenn man in das Wesen der Wissenskultur des Alten Orients eindringen möchte. Verharren wir also bei dem eigentümlichen Phänomen, dass mesopotamische Gelehrte Daten und Erkenntnisse sammelten und systematisierten, aber nie die den Daten innewohnenden Prinzipien in abstrakten Lehrsätzen und Gesetzen formulierten.

In den mathematischen Texten sammelten sie anstelle von Beweisen und Lehrsätzen Aufgaben desselben Typs mit geringfügigen Abweichungen, um ein Problem von verschiedenen Seiten zu beleuchten. Vergleichbares ist für alle weiteren Wissensbereiche zu verzeichnen. So wurden für das Studium der sumerischen Sprache zahlreiche Paradigmata angelegt, ohne dass eine Grammatik formuliert worden wäre. In Hunderttausenden von Omina werden zwar Protasis ${ }^{14}$ und Apodosis ${ }^{15}$ in einen kausalen Zusammenhang gestellt. Aber Lehrbücher, die die zugehörige Hermeneutik liefern, fehlen vollständig (sieht man einmal von den Kommentaren ab). Im Kodex Hammurapi und in anderen Rechtsbüchern finden wir Zusammenstellungen von Rechtssprüchen, die keineswegs alle Bereiche des Rechtes abdecken. Die Rechtsnormen, die den Rechtssprüchen und aller richterlicher Tätigkeit letztlich zugrunde liegen, sind jedoch nirgendwo schriftlich fixiert. Sie offenbaren sich

\footnotetext{
12 v. Soden, Einführung in die Altorientalistik, S. 163.

13 Pichot, Geburt der Wissenschaft, S. 124f.

14 Protasis wird in der Omenlehre der Satz genannt, in dem das beobachtete Vorzeichen beschrieben ist.

15 Apodosis heißt der auf die Protasis folgende Satz, in dem das mit dem Vorzeichen verknüpfte, in der Zu-

kunft stattfindende Ereignis beschrieben ist.
} 
erst demjenigen, der die paradigmatischen Rechtssprüche des großen Gerechten der mesopotamischen Kultur immer wieder studiert. Aus diesem Grunde wurde der Kodex Hammurapi immer wieder abgeschrieben, der Text immer wieder auswendig gelernt und wohl auch immer wieder im Kreise der Gelehrten diskutiert. Abschreiben und Auswendiglernen, Auswendiglernen und Aufschreiben, so zeigt es auch die Didaktik mesopotamischer Schultexte, sollten den Adepten der Wissenschaft in den Gegenstand seiner Disziplin einführen. „Mit Tafel und Griffel“ (so hieß es in dem oben zitierten Enmeduranki-Text) sollte er die zugrunde liegenden Lehren und Erkenntnisse tastend und angeleitet durch die ihm im Studienmaterial bereitgestellten Exempla erspüren und durch stetiges Studium in sich immer deutlicher erstehen lassen. Unter diesem Gesichtspunkt lässt sich im übrigen auch verstehen, warum alle sog. Fachbibliotheken mesopotamischer Gelehrter fast ausschließlich aus Tontafelabschriften bestehen, die junge, angehende Gelehrte anfertigten. Die erst durch ein dauerhaftes Studium erkannten grundlegenden Weisheiten zu formulieren und so den Lernenden zugänglich zu machen, hätte, so will mir scheinen, in der mesopotamischen Wissenskultur bedeutet, das Ziel vor den Weg zu stellen. Sollte sich also in der mesopotamischen „Theorieverweigerung" etwa doch nicht mangelndes Abstraktionsvermögen, sondern eine sich von der unseren grundlegend unterscheidende Wissenskultur offenbaren, die den Vorteil gehabt hätte, jeden Gelehrten mit den Grundlagen seines Tuns ringen zu lassen, um diese umso besser kennen zu lernen? Wir sollten darüber nachdenken, ob den Gelehrten Mesopotamiens das Formulieren von Lehrsätzen nicht als ein letztlich schädliches Banalisieren des eigentlich Unaussprechlichen erschienen ist. In dem oben zitierten Enmeduranki-Text war der Gelehrte als derjenige charakterisiert, der „das Geheimnis der großen Götter bewahrt". Das unaussprechliche und im Uranfang von den Göttern gegebene Gesetz, das unsere Kultur in Lehrsätzen zu fassen versucht, ist, so meine ich, das, was die Babylonier als nisirti ilani rabuti bezeichneten, das unaussprechliche „Geheimnis der großen Götter“, das aller Wissenschaft zugrunde liegt.

Aus der jüdischen Kultur ist uns Vergleichbares bekannt. Mit dem Ziel, das Unfassbare der Weltenschöpfung fassbar zu machen, das Unaussprechliche in sprachliche Form zu bringen, hat der Redaktor der Thora gleich zwei sich grundlegend widersprechende Schöpfungsberichte nebeneinandergestellt, obgleich beide als das Wort des einen Gottes gelten. Während das Judentum in guter altorientalischer Tradition die auf der Oberfläche sichtbaren Widersprüche als unerschöpflichen Quell der in Studium und Gelehrtengespräch entfalteten Erkenntnis nutzt, kapriziert sich die moderne westliche Rezeption mit ihrem „digitalen Denken“ darauf, die Widersprüche durch Quellenscheidung aus dem Weg zu räumen.

Hiermit dürfte die christliche Bibelexegese der jüdischen Kultur der Bibelauslegung ebenso wenig gerecht werden wie die moderne Wissenschaftsgeschichte der Wissenskultur des Alten Orients. 
Blättert man in den gängigen modernen Wissenschaftsgeschichten, so entdecken die Wissenschaftshistoriker in Mesopotamien eine „erwachende Wissenschaft" in den Bereichen der Mathematik, der Astronomie und der Medizin. Bezeichnenderweise fehlen Darstellungen über die Philologie, die Theologie und die nichtmedizinischen Therapieformen, die offenbar ebenso wenig "wissenschaftsverdächtig" sind wie Historiographie oder gar Divination. Auch hier gilt das bereits zuvor Gesagte. Wenn man so aus dem Blickwinkel des modernen europäisch-westlichen Wissenschaftsverständnisses auf die Gelehrsamkeit einer fernen und fremden Kultur schaut, wird man wenig über diese andere Kultur erfahren können. Über die zweifelhafte Erkenntnis, dass diese ferne Kultur zwar wenige, aber immerhin einige erste mehr oder weniger unbeholfene Schritte auf einem Weg eingeschlagen hat, den die eigene Kultur zielstrebig gegangen ist, wird man nie hinauskommen. Darum schieben wir diesen wenig fruchtbar erscheinenden Blickwinkel einfach beiseite. Denn André Pichots Urteil, , dass [in Mesopotamien] die Vernunft noch nicht zum obersten Wahrheitskriterium erhoben worden ist ${ }^{\text {"16 }}$ (dies sollten ja bekanntlich erst die Griechen leisten), wird uns dem Verständnis mesopotamischer Wissenschaft nicht näher bringen können. Dies sei anhand eines Beispiels aus dem Bereich der babylonischen Heilkunde deutlich gemacht. In einer Therapiebeschreibung, die im 7. Jh. in Assur niedergeschrieben wurde, aber erheblich ältere Vorläufer besitzt, sind die Symptome einer Krankheit aufgeführt, die, wenn sie unbehandelt bleibt, zum Tode führt. Ich zitiere:

Wenn einem Mann ein ungutes Vorzeichen erscheint (...) und er beständig und immer wieder Verlust und Abgang erleidet; (er) einen Abgang an (den Zahlungsmitteln) Gerste und Silber (erfährt); (er) einen Abgang an (den Arbeitskräften) Knecht und Magd (erfährt); (wenn) Rinder, Pferde und Kleinvieh, Hunde, Schweine und Menschen gleichermaßen immer wieder sterben (und) er immer wieder sein Selbstvertrauen verliert; er Anweisung gibt, ohne dass dem willfahren wird; er ruft, ohne dass geantwortet wird; er sich dem Begehren, das die Leute formulieren, bereitstellt; (wenn) er in seinem Bett immer wieder in Schrecken gerät (und) Lähmungszustände bekommt; seine Gliedmassen immer wieder , hingeschüttet' sind (und) er dann und wann erschrickt; (wenn) er bei Tage und bei Nacht nicht schlafen kann; (wenn) er immer wieder schreckliche Träume sieht; (wenn) er, während er kaum zu essen und zu trinken vermag, das, was er sagt, (gleich) wieder vergisst.

Was diesen Mann anbetrifft: Der Zorn von Gott und Göttin ist ihm immer wieder auferlegt (...).

${ }^{16}$ Pichot, Geburt der Wissenschaft, S. 145. 
Für diesen Mann (gilt): (?): an der „Hand des Bannes“, der „Hand des Gottes“, der „Hand der Menschheit", der „Krankheit des Zusammengewehten“ ist er erkrankt.

Die Schuldenlasten des Vaters und der Mutter, des Bruders und der Schwester, der Familie, des Geschlechtes und der Sippe packten ihn.

Um (dies) zu lösen, so dass die Verfinsterungen, (die) ihn (betreffen), nicht mit (schlimmeren Folgen) an ihn herankommen können.

Es würde zu weit führen, in alle Einzelheiten zu gehen. Eines aber ist klar: Der Rahmen dessen, was wir Medizin nennen, ist hier bei weitem überschritten. Die Krankheitssymptome werden keineswegs nur am Körper des Kranken erkannt. Die Babylonier sehen die Krankheit schon mit einem Vorzeichen in den Lebensbereich des Menschen einbrechen und dann immer engere Kreise um den, Infizierten' ziehen. Erst sind die Symptome nur ökonomischer Natur. Dann gehen Geld und Arbeitskräfte nicht nur verloren, sondern Mensch und Tier sterben im Umkreise des Betroffenen. Daraufhin schwinden Selbstvertrauen und Autorität des Betroffenen. Und schließlich kommt es zu ersten physischen Symptomen, denen auch wir medizinische Natur zusprechen würden. Obgleich die Babylonier wussten, dass die Krankheit übertragen werden konnte (wie zum Beispiel durch das Trinken aus dem Becher eines Unreinen) und danach noch Zeit ins Land ging, bis sie ausbrach, kam für sie die Übertragung nicht durch Unachtsamkeit oder gar einen unglücklichen Zufall zustande, sondern auf Beschluss der Götter, die wegen lange ungeahndet gebliebener Vergehen von Vorfahren oder Familienmitgliedern verärgert waren.

Haben wir es wirklich mit einer Krankheit zu tun? Aus dem Blickwinkel der modernen Medizin macht sich ratloses Unverständnis breit. War die Therapie der Babylonier ein Fall für den Wahrsager, den Priester, den Magier, den Medizinmann oder für den Arzt, den Psychologen oder gar den Vermögensberater? Der rührende Versuch der Assyriologie, die nicht in Frage zu stellende Leistungsfähigkeit mesopotamischer Heilkunde dadurch für sich und den modernen Zeitgenossen verdaulicher zu machen, dass sie (übrigens in einem Zirkelschluss) die Heilkunde Mesopotamiens in den Verantwortungsbereich eines vorwissenschaftlich arbeitenden Beschwörers und in den eines rational, mit pharmakologisch wirksamen Mitteln arbeitenden Arztes einteilt, ist nicht haltbar. So unbequem und bedrohlich es auch erscheinen mag: der Heiler, der um die auch uns einsichtige Wirkkraft von Heilkräutern genau weiß und sie als Medizinen einsetzt, ist derselbe, der zu Beginn der Therapie die Götter mit Opfer und Gebet besänftigt; der das zurückliegende Vergehen der Vorväter durch Figurenzauber ungeschehen macht; der in einem magischen Gerichtsverfahren den Omenanzeiger des unrechtmäßigen Zugriffs auf den Patienten beschuldigt und ihn mit Hilfe der Götter verurteilt. Er ist es, der nach den strengen Regeln göttlicher Offenbarung die keimende Krankheit von dem Patienten mit Getreideschrot herunterreibt und die Rückstände ver- 
brennt und dann die Krankheit mit dem sakramentalen Segen der Götter in ein Tonfigürchen bannt, um sie nun doch noch mit dem Patienten zu vermählen: denn der der Krankheit zugrunde liegende Beschluss der Götter, den Patienten zu strafen, kann nicht rückgängig gemacht werden. Er muss sich vollziehen, wenngleich auch nur an einem Abbild des Patienten, auf das dessen Identität übertragen wurde. Erst jetzt kann der so dem Gottesurteil entgangene Patient mit den Mitteln behandelt werden, die wir annähernd medizinisch nennen.

In übersichtlich aufgebauten, sehr umfangreichen (und immer noch nicht erschlossenen) keilschriftlichen Nachschlagewerken auf Tontafeln konnte sich der Heiler über Aussehen und Heilwirkung von Pflanzen und Mineralien informieren. Diese Werke muten in ihrem rationalen Aufbau und mit den exakten Beschreibungen sehr modern an. Und in der Tat kann die Wirkkraft mancher Heilverfahren und Heilmittel auch von der modernen Medizin nachvollzogen werden. Gleichwohl ist auch in diesen Fällen vor einem vorschnellen und vielleicht nur vermeintlichen Gefühl der Vertrautheit zu warnen. Denn auch die nach der Einschätzung der modernen europäischen Medizin wirksamen mesopotamischen Medikamente galten keineswegs als aus sich selbst heraus wirkkräftig. Erst ein sakramentaler Segen konnte ihnen diese Kraft verleihen. In wichtigen Fällen, etwa dann wenn dem König selbst eine Medizin verabreicht werden sollte, erschien es den mesopotamischen Ärzten ratsam, die Wirksamkeit der Medizin zu testen. Dies geschah weder im klinischen, noch im Tier- oder im Selbstversuch. Man holte mittels Leberschau die Meinung der Götter ein.

Spätestens an dieser Stelle wird, so hoffe ich, offenbar, dass ein Projizieren unserer zeit- und kulturgebundenen Wissenschaftsdisziplinen auf die Heilkunde des Alten Orients oder auf andere Wissensbereiche nur zu unzulässiger selektiver Wahrnehmung oder zu hoffnungslos irreführenden Zerrbildern führen würde.

Auf einen weiteren, m. E. sehr wichtigen Punkt gilt es noch hinzuweisen. Um zu einem tieferen Einblick in die mesopotamische Wissenskultur zu gelangen, ist eine ernsthafte Beschäftigung mit den mesopotamischen Vorstellungen von Wesen und Wirkkraft der Materie vonnöten. An anderer Stelle ${ }^{17}$ habe ich versucht aufzuzeigen, worin Heilkraft und magische Wirkung dreier, in Heilungs- und Reinigungsritualen sehr häufig und fast immer gemeinsam verwendeter Pflanzen und Pflanzenteile besteht, nämlich binu, Tamariske; maschtakal, ein alkalihaltiges, für die Herstellung einer Seifenlauge geeigne-

${ }^{17}$ Maul, Zukunftsbewältigung, S. $62 \mathrm{ff}$. 
tes Kraut; und libbi gischimmari, der, Vegetationskegel der Dattelpalmé. Dies soll hier nicht im einzelnen nachvollzogen werden. So viel sei jedoch gesagt. Eine jede der drei Pflanzen steht für eine klar definierte Phase in der Reinigung und Heilung eines Patienten. Die Tamariske (binu) hatte die Aufgabe, das Voranschreiten des „Bösen“, das von einem Menschen oder auch einer Sache Besitz zu ergreifen begann, so zu verhindern oder genauer abzubrechen, wie ,eine [einmal] ausgerissene Tamariske nicht mehr an ihren ursprünglichen Ort zurückkehren kann und auf ihr Früchte nicht mehr wachsen können ${ }^{{ }^{18} 8}$. Das Seifenkraut maschtakal stand dafür, dass das Böse von dem Betroffenen „heruntergewaschen“ wurde. Und der Vegetationskegel der Dattelpalme (libbi gischimmari) schließlich, als „Motor“, als treibenden Kraft der Pflanze, der (das südliche) Mesopotamien seine Lebensgrundlage und seinen Reichtum verdankt, verkörperte das Sich-segensreich-Entwickeln, in dessen Genuss der Patient gelangen sollte. Gemeinsam verwendet stehen sie nicht nur für den sich aus den soeben benannten drei Phasen zusammensetzenden Prozess des Heil-, des Reinwerdens, sondern tragen ihn in sich, bewirken ihn. Demnach trägt die leblose, statische Materie (jedenfalls der assyrisch-babylonischen Anschauung zufolge) die Dynamik eines Ablaufes in sich und vermag diese freizusetzen oder hervorzurufen. Wer an dieser Stelle nicht folgen mag, lässt sich vielleicht durch ein anderes Beispiel dem vorgetragenen Gedanken geneigter machen: aus einem jüngst publizierten Text wissen wir, dass ein apotropäisches Figürchen, eingesetzt zum magischen Schutz des Hauses, damit es wahrhaft wirksam sei, nicht nur aus mit Wasser geschmeidig gemachtem Ton gefertigt werden sollte, sondern der Ton sollte mit me qiddati aufbereitet werden, mit „abwärts fließendem Wasser". Dieses aber, nach unserer modernen Sicht der Dinge, ist ebenso gut oder schlecht geeignet zur Herstellung einer Statuette wie etwa das stehende Wasser einer Zisterne. Für den altorientalischen Menschen aber trägt es die (in Gebeten und Ritualen häufig namentlich genannte) Kraft des Wassers in sich, das Schmutz und Verunreinigung nicht nur abwäscht, sondern so unwiederbringlich abtransportiert, wie - so vermerkt ein einschlägiger Text - „das flussabwärts fließende Wasser nicht zurück flussaufwärts fließen kann". ${ }^{9}$

Spätestens an dieser Stelle zeigt sich, - ohne dass wir hier den einzelnen Beispielen weiter nachgehen könnten oder müssten - dass sich die assyrischbabylonischen Vorstellungen von der Materie und ihrer Wirkkraft sehr deutlich von den unseren unterscheiden und ein Studium sog. ,esoterischer" Keilschrifttexte für ein tieferes Verständnis der Kulturgeschichte und der Archäologie des Alten Orients nicht nur lohnenswert, sondern unerlässlich ist.

\footnotetext{
18 Siehe ebd., S. 65.

19 Ebd., S. 88 f.
} 
Wir sind, man muss es leider zugeben, sehr weit davon entfernt, auch nur ansatzweise das komplexe Geflecht von Bezügen zu verstehen, das in den zahllosen Keilschrifttexten ganz unterschiedlicher Gattungen offensichtlich oder versteckt zwischen Farben, Pflanzen, Tieren, Steinen, Mineralien und Metallen, Körperteilen und Planeten, Göttern sowie irdischen und kosmischen Bereichen und Kräften aufgestellt wird. Die sogenannten esoterischen Listen liefern uns hierzu einen Schlüssel. Da erscheinen Metalle als Emanationen von Göttern, Pflanzen haben ihre Entsprechungen im Tierreich und Körperteile sind Monaten zugeordnet. Der Hintergrund dieser Lehren liegt noch weitestgehend im Dunkeln und ist in Theorien und Lehrsätzen nie formuliert worden. Ihn zu erforschen, wird uns zweifelsohne dem Wesen mesopotamischer Gelehrsamkeit bedeutend näher bringen.

Schon jetzt wissen wir, dass die ggf. unklare Bedeutung bestimmter beobachteter astronomischer Befunde durch Leber- und Eingeweideschau verifiziert werden konnte und dass, wie uns ein „Handbuch eines babylonischen Sehers" übermittelt, die astronomischen Befunde nicht wirklich aussagekräftig waren ohne genaueste Beobachtungen der terrestrischen Zeichen, die als Spiegel und Gegenstück der himmlischen galten. In dem einen, dem astronomischen, spiegelte sich das andere System, das der terrestrischen Zeichen, das seinerseits in dem System der Lebertopographie eine weitere Emanation fand. Auf Lebermodellen wiederum finden wir bestimmten Leberteilen Himmelsregionen zugewiesen. All dies zeigt deutlich, dass die Babylonier von dem Gedanken getragen waren, dass in der von ihnen erfahrbaren materiellen Welt alle Dinge mit allen Dingen in Verbindung standen und jeweils nur unterschiedliche Emanationen des Einen waren: Ausdrucksformen der nicht benennbaren und sprachlich nicht fassbaren göttlichen Ordnung, die mit einem eigenen Willen den Bereich des Irdisch-Menschlichen leitete. Ein wesentlicher Teil der "wissenschaftlichen Energie“ des Alten Orients wurde in die „Entdeckung“ dieses Bezugssystems, des „Bandes zwischen allen Dingen“ investiert, ein Bezugssystem, in dem sich das eine durch das andere offenbart. Dieses dem europäisch-westlichen Denken völlig fremde Erkennen des einen im anderen ist der Gelehrtentradition Mesopotamiens durchaus angemessen. Denn die Keilschriftgelehrten waren es von Kindheit an gewohnt, eine Schrift zu beherrschen, in der Keilschriftzeichen grundsätzlich vieldeutig waren und neben ihrem intendierten, jeweils kontextbezogenen Sinn immer die übrigen potentiellen Bedeutungen virtuell mittrugen. Über viele Jahrhunderte nahmen sie die beiden Sprachen (das Sumerische und das Akkadische), in denen sie schrieben und dachten, keineswegs als zwei gänzlich unverwandte Sprachen wahr, sondern als zwei analoge Emanationen von einer Sprache, die selbst sich dem Sprachlichen entzog und somit ein Geheimnis blieb. 
Auch das Erforschen der Zeichenhaftigkeit der Welt stand für die Gelehrten Mesopotamiens im Dienste der Divination, die heute als übler Aberglaube gilt. Dies mag sein. Über einer solchen Wertung wird allerdings allzu leicht vergessen, dass im Alten Orient mit der Divination eine Idee Gestalt annahm, die - in bisweilen fataler Weise - unsere Gesellschaft bis heute bestimmt: nämlich die Vorstellung, dass die gesamte Welt einem Gefüge von strengen Gesetzmäßigkeiten unterworfen sei, die es nur zu erkennen gilt, um dann sich ihrer bedienend - die Welt in Harmonie lenken zu können. Nichts anderes bezweckt schließlich moderne Wissenschaft. Der großangelegte Versuch der altorientalischen Kulturen, mit der im Omen durch die Verknüpfung von Protasis und Apodosis erkannten Kausalität Gesetzmäßigkeiten im historischen Geschehen zu ermitteln, um diese dann für das eigene politische Handeln nutzbar zu machen, findet in seiner Kühnheit doch nicht einmal im Historischen Materialismus sein Gegenstück!

Wenn wir Werte, Kategorien und Leistungen einer fremden Kultur erschließen möchten, gelingt dies nur, wenn wir die eigenen Werte und Kategorien nicht zum Maß aller Dinge machen. Nur ehrfürchtiger Respekt, Offenheit, genaues Hinsehen und große Sachkenntnis werden uns hier weiterbringen. Sind wir, dies zu leisten, nicht bereit, wird es uns ergehen, wie dem Fuchs in der folgenden Geschichte:

Gevatter Fuchs hat einst in Kosten sich gestürzt und den Gevatter Storch zum Mittagbrot gebeten. Nicht allzu üppig war das Mahl und reich gewürzt; denn statt der Austern und Lampreten gab's klare Brühe nur - viel ging bei ihm nicht drauf.

In flacher Schüssel ward die Brühe aufgetragen; indes Langschnabel Storch kein Bisschen in den Magen bekam, schleckt Reineke, der Schelm, das Ganze auf.

Doch etwas später lädt der Storch, aus Rache

für diesen Streich, den Fuchs zum Mahle auf seinem Dache. „Gern", spricht Herr Reineke, „da ich nach gutem Brauch mit Freunden nie Umstände mache."

Die Stunde kommt; es eilt der list'ge Gauch nach seines Gastfreunds hohem Neste, lobt dessen Höflichkeit aufs beste, findet das Mahl auch schon bereit, hat Hunger - diesen hat ein Fuchs zu jeder Zeit -, und schnüffelnd atmet er des Bratens Wohlgerüche, des leckren, die so süß ihm duften aus der Küche. Man trägt ihn auf, doch - welche Pein! in Krügen eingepresst, langhalsigen und engen; leicht durch die Mündung geht des Storches Schnabel ein, 


\title{
umsonst sucht Reineke die Schnauze durchzuzwängen. Hungrig geht er nach Haus und mit gesenktem Haupt, klemmt ein den Schwanz, als hätt' ein Huhn den Fuchs geraubt, und lässt vor Scham sich lang nicht sehen.
}

\author{
Ihr Schelme, merkt euch das und glaubt: \\ Ganz ebenso wird's euch ergehen. ${ }^{20}$
}

\section{Literatur}

Burstein SM (1978) The Babyloniaca of Berossus. In: Sources from the Ancient Near East $1 / 5: 143-181$

Kuhn D, Stahl H (Hrsg.) (2001) Die Gegenwart des Altertums. Formen und Funktionen des Altertumsbezugs in den Hochkulturen der Alten Welt, Heidelberg: Edition Forum

Lambert WG (1998) The Qualifications of Babylonian Diviners. In: Maul SM (Hrsg.) Festschrift für Rykle Borger zu seinem 65. Geburtstag am 24. Mai 1994. tikip santakki mala baschmu .... Cuneiform Monographs 10, Groningen: Styx, 141-158

Maul SM (1994) Zukunftsbewältigung. Eine Untersuchung altorientalischen Denkens anhand der babylonisch-assyrischen Löserituale (Namburbi). Baghdader Forschungen, Bd. 18. Mainz: Zabern

Pichot A (1995) Die Geburt der Wissenschaft, Darmstadt: Wissenschaftliche Buchgesellschaft Schnabel P (1923) Berossos und die babylonisch-hellenistische Literatur. Leipzig: Teubner Sjöberg A (1971/72) In Praise of the Scribal Art. In: Journal of Cuneiform Studies 24:126-131 v. Soden W (1985) Einführung in die Altorientalistik. Darmstadt: Wissenschaftliche Buchgesellschaft

${ }^{20}$ Jean de La Fontaine, Die Fabeln. Gesamtausgabe in deutscher und französischer Sprache, mit über 300 Illustrationen von Gustave Doré (aus dem Französischen übersetzt von Ernst Dohm), Wiesbaden o. J. [1978], S. 29.

Heidelberger Jahrbuch, Band 47 (2003) H. Gebhardt/H. Kiesel (Hrsg.): Weltbilder

c) Springer-Verlag Berlin Heidelberg 2004 